ARTíCUlos De INVESTIGACIÓN

\title{
La enseñanza del profesionalismo a estudiantes de licenciatura en Derecho en México: Incidencia en el ejercicio de la profesión jurídica
}

\author{
Ensino de profissionalismo a estudantes de Direito no México: \\ O impacto sobre o exercício da profissão de advogado \\ Teaching of professionalism to undergraduate students of Law in Mexico: \\ The impact on the practice of the legal profession
}

\author{
Yurixhi Gallardo iD y Montserrat Gómez Villaseñor iD \\ Universidad Panamericana, México
}

\begin{abstract}
RESUMEN El profesionalismo es un elemento clave de la ética profesional. La investigación aborda el profesionalismo como una competencia que requerirán los estudiantes de Derecho en México. Este trabajo está dividido en tres apartados: el primero explica qué se entiende por profesionalismo y su vínculo con la ética profesional; el segundo aborda la enseñanza del profesionalismo; y en el tercero se analizan los resultados de una autoevaluación realizada por alumnos de primer año de licenciatura en Derecho en una universidad del occidente de México. El estudio muestra, por una parte, que el profesionalismo es un elemento de la ética profesional que necesita ser enseñado y, por lo tanto, abordado como competencia profesional. Por otra parte, muestra los resultados de una autoevaluación de profesionalismo realizada por estudiantes. Dicha autoevaluación aparece como un primer paso para el desarrollo del profesionalismo como competencia profesional.
\end{abstract}

PALABRAS CLAVE Competencia profesional, educación jurídica, educación superior, ética profesional y profesionalismo.

RESUMO Profissionalismo é um elemento chave da ética profissional. A pesquisa aborda o profissionalismo como uma competência que será exigida pelos estudantes de direito no México. Este trabalho está dividido em três seções: a primeira explica o que significa profissionalismo e sua ligação com a ética profissional; a segunda trata do ensino do profissionalismo; finalmente, são analisados os resultados de uma auto-avaliação realizada por estudantes de direito do primeiro ano em uma universidade no oeste do 
México. O estudo mostra, por um lado, que o profissionalismo é um elemento de ética profissional que precisa ser ensinado e, portanto, tratado como uma competência profissional. Por outro lado, mostra os resultados de uma auto-avaliação do profissionalismo realizada pelos estudantes. Esta auto-avaliação aparece como um primeiro passo para o desenvolvimento do profissionalismo como uma competência profissional.

PALAVRAS-CHAVE Competência profissional, educação jurídica, educação superior, ética profissional e profissionalismo.

ABSTRACT Professionalism is a key element of professional ethics. The paper approaches professionalism as a competence that students of Law in Mexico will require. The work is divided into three sections: the firt explains what is meant by professionalism and its link with professional ethics; the second part deals with the teaching of professionalism; finally, it analyzes the results of a self-evaluation carried out by first-year law students at a university in western Mexico. The study shows that professionalism is an element of professional ethics that needs to be taught and therefore proposes to approach it as professional competence. On the other hand, it shows the results of the self-assessment in professionalism carried out by students. Self-evaluation appears as a first step in the development of professionalism as a professional competence.

KEYWORDS Professional competencies, legal education, higher education, professional ethics and professionalism.

\section{Introducción}

En lo que respecta a la profesión jurídica en México, el artículo 100 de la Constitución Política de los Estados Unidos Mexicanos, en el párrafo séptimo, señala que el profesionalismo es uno de los principios bajo los cuales se regirá la carrera judicial. Dicho término es utilizado como principio de actuación de ciertos funcionarios públicos. El texto constitucional se refiere al profesionalismo con respecto a la actuación de los servidores públicos de la fiscalía en el artículo 102, o de la procuración de justicia en la Ciudad de México o en los Estados en los artículos 122 A y 116, respectivamente. Además, hace referencia a los principios de actuación que han de regir en los centros de conciliación en el ámbito local y federal en el artículo 123, a los principios que rigen el funcionamiento del Instituto Federal de Acceso a la Información en el artículo 6 y a la actuación de las instituciones de seguridad pública en el artículo 21.

El profesionalismo resulta de tal relevancia por mandato constitucional para el ejercicio de la función judicial que el Código de Ética del Poder Judicial de la Federación desglosa las acciones en las que se concreta. La doctrina en México no ha estudiado ampliamente el término como elemento de la ética jurídica. Saldaña (2014) sostiene que «el profesionalismo es una cualidad que distingue a la persona cuando 
esta lleva a efecto su labor o actividad de manera relevante, con una mayor capacidad y aplicación que otras personas que realizan la misma acción». Por lo tanto, es necesario conocer los alcances del profesionalismo en la profesión jurídica toda vez que es un mandato constitucional para la impartición de justicia.

Aunque en México poco se ha estudiado el término de acuerdo a Saldaña (2014), el profesionalismo es un concepto que tiene una larga historia y relevancia en su tratamiento. La preocupación acerca del profesionalismo es tan antigua como las profesiones (Hession, 2019). Como señala Evetts (2013), en el campo de la sociología de las profesiones, su estudio posee una larga historia. Sin embargo, la crisis que enfrentan diferentes profesiones, entre ellas el derecho, ha llevado a afirmar que también el profesionalismo enfrenta una crisis (Hession, 2019). Bower y Burmeister (2013) sugieren que el profesionalismo debería estar posicionado de manera que fuera comercialmente deseable que los profesionales se comportaran de acuerdo con un código de ética. Por su parte, en Estados Unidos y en otros países el tema ha sido ampliamente estudiado en distintas profesiones, incluido el derecho (Bagust, 2013; Francis, 2005; O'Sullivan y otros, 2012). En este sentido, ha jugado un papel importante para el estudio de este concepto el que sea considerado como uno de los resultados de aprendizaje para las escuelas de Derecho en Estados Unidos, y también es relevante mencionar la importancia que ha cobrado la evaluación de tales resultados (Wegner, 2018). Entre los autores que han abordado el profesionalismo ampliamente dentro de la profesión jurídica destaca Hamilton (2017a, 2017b).

En México, el profesionalismo al que hace referencia el texto constitucional, a propósito de la carrera judicial, invoca una de las competencias de los profesionales del derecho que no es citada en los códigos de ética de los profesionales. ${ }^{1}$ Supone un reto que los profesionales se comporten de acuerdo con lo que la máxima norma del país señala si no están familiarizados con el término.

Por otra parte, resulta urgente que el desarrollo de modelos pedagógicos por competencias haga referencia al profesionalismo. Sin embargo, la mayoría de las escuelas de derecho no consideran los modelos pedagógicos por competencias (González Ramos, 2017). De ahí la urgente necesidad de abordar dicha competencia con los estudiantes desde el inicio de los estudios de la licenciatura en Derecho. Ya la literatura académica latinoamericana ha restreado cómo los modelos de educación jurídica por competencias son una respuesta para la formación de los profesionales acorde a las necesidades actuales (Olson y Lazaretti, 2019).

En este sentido, el objetivo de este trabajo es mostrar al profesionalismo como

1. Asociación Nacional de Abogados de Empresa Colegio de Abogados, «Código de Ética», disponible en https://bit.ly/3mGejef; Barra Mexicana, Colegio de Abogados, «Código de Ética Profesional», 2017, disponible en https://bit.ly/3pxSmQr. Ilustre y Nacional Colegio de Abogados de México, «Código de Ética del Ilustre y Nacional Colegio de Abogados de México», 1997, disponible en https://bit.ly/32W41GO. 
una competencia, sosteniendo la necesidad de enseñarla y evaluarla. En este sentido, la competencia de profesionalismo, por un lado, es una competencia que requiere de saberes declarativos y, por otro lado, es actitudinal; sumado a lo anterior también puede ser considerada procedimental. Por lo tanto, presentamos los resultados de la autoevaluación realizada por estudiantes de primer año de licenciatura en ciertos aspectos que conforman el profesionalismo. La pregunta que orientó la investigación fue: ¿cómo pueden los estudiantes de primer año de la licenciatura en Derecho conocer el grado de profesionalismo en el que se encuentran? A partir de dicho planteamiento se desarrolló la investigación, consciente de que el profesionalismo en la carrera judicial y en otros ámbitos, como ha sido señalado, es en México un mandato constitucional.

El trabajo se divide en tres partes. En el primer apartado se aborda el concepto de profesionalismo (Hamilton, 2017a; Moorhead, 2014). En la segunda parte se analiza la necesidad de la enseñanza del profesionalismo como competencia profesional y la necesidad de su evaluación. En el tercer apartado se presentan los resultados de una autoevaluación realizada por estudiantes de primer año de la licenciatura en Derecho a través de una rúbrica.

La investigación es una de tipo mixta que se llevó a cabo de manera documental y comparativa. De esta forma, se elaboró una síntesis bibliográfica como método de investigación para la primera y para la segunda parte del trabajo, y se compara la aplicación del concepto en el derecho frente a la medicina. Se profundizó en el concepto de profesionalismo, así como en la enseñanza de este como competencia profesional. Sumado a esto, se estudia empíricamente el profesionalismo. Siendo así, en el tercer apartado, 52 estudiantes de primer año de la licenciatura en Derecho de una universidad del occidente de México se autoevaluaron con una rúbrica desarrollada por el Holloran Center for Ethical Leadership in the Professions a través del proyecto Milestone (Holloran Center for Ethical Leadership in the Professions, 2019). Dicho proyecto consiste en el desarrollo de rúbricas que evalúan competencias asociadas con los resultados de aprendizaje requeridos para las escuelas de Derecho en Estados Unidos. Una futura investigación podría utilizar de referencia las competencias propuestas a partir del Proyecto Tuning, en el que aparece el compromiso ético como una competencia interpersonal y, a partir de ella, estudiar el profesionalismo (Aguado y otros, 2017). La investigación bibliográfica no arrojó ejercicios similares en América Latina, lo que puede deberse a la poca comprensión del término, como será explicado en la investigación. Aunque en otros rubros hay ejercicios de evaluación de competencias éticas en aspectos específicos, por ejemplo, el género (Bas y otros, 2017).

El trabajo concluye con la mención de la urgente necesidad de profundizar en la comprensión del profesionalismo como una competencia profesional en México, así como la necesidad de enseñarlo y evaluarlo durante los años de educación universitaria, para así lograr lo que dispone el texto constitucional. No puede caerse en el error 
de pensar que dicha competencia podrá ser adquirida por los estudiantes a través de un curso único de ética, cuando ya existe investigación que demuestra lo contrario (Hamilton, 2012). Por otra parte, se propone que a los estudiantes, desde el inicio de la licenciatura, se les ofrezcan herramientas que les permitan familiarizarse con el alcance del profesionalismo.

\section{Profesionalismo y ética profesional}

El profesionalismo es una noción compleja que ha sido abordada, en primer lugar, desde la sociología de las profesiones. Taylor, Grey y Checkland (2017) advierten que la literatura sociológica se enfoca en el contrato social, en el servicio público, en los roles, en la identidad y en el poder, mientras que la literatura educativa destaca los comportamientos, las actitudes y los rasgos. Por su parte, Evetts (2013) sintetiza el camino ideológico que ha recorrido tal concepto hasta ser vinculado con los códigos de ética profesional que permiten orientar las conductas de los profesionales. En la configuración del profesionalismo están involucrados distintos actores, entre ellos las escuelas, las culturas de trabajo y quienes ejercen la profesión, entre otros.

Así, la sociología ha estudiado el profesionalismo a través de las profesiones destacando que los controles internos y externos de la profesión se justifican a través de este. El concepto de profesionalismo resulta atractivo para los practicantes, empleados y gerentes en el desarrollo y mantenimiento de las identidades en el trabajo. El discurso del profesionalismo es un poderoso instrumento para el cambio ocupacional y control social en niveles macro, mega y micro (Evetts, 2013).

La literatura legal y médica han examinado ampliamente el concepto de profesionalismo (Frye y otros, 2020; Hamilton, 2017a; Hernández y otros, 2010; Hession 2019). Por lo que concierne a otras profesiones, el concepto es poco explorado, entre otras razones, por la poca investigación acerca de la profesión. Por ejemplo, con respecto a las áreas de la salud, ha quedado de manifiesto que cada país considera al profesionalismo con una ligera variación (Cruess y Cruess, 2016). Por otra parte, también en la medicina hay voces críticas del profesionalismo que señalan que, en la práctica, es una herramienta de control social usada por determinados grupos para mantener el dominio sobre la medicina y su autorregulación (Frye y otros, 2020). En lo que hay consenso es que el profesionalismo hace referencia a valores, conductas, moralidad e identidad (Victoria y otros, 2020). Por su parte, Sylvia y Richard Cruess (2016) hacen referencia al profesionalismo cuando se piensa, actúa y se siente como médico, lo cual varía de país en país y, por lo tanto, dichas diferencias deben ser respetadas. En el área médica, el profesionalismo tiene tal relevancia que ha sido incluido como una de las competencias centrales cuya evaluación es necesaria para poder graduarse (Sullivan y otros, 2007). Por consecuencia, la literatura al respecto, en el ámbito médico, es extensa. 
Por lo que concierne al derecho, resulta aplicable lo que señalan Cruess y Cruess (2016) con respecto al profesionalismo en la medicina, es decir, pensar, actuar y sentir como abogado presenta variaciones en diversos países, sin embargo, es posible identificar rasgos comunes. Desde el derecho, una primera aproximación al profesionalismo remite a la perspectiva filosófica o a las reglas de moral. Sullivan sostiene que la identidad profesional engloba tradicionalmente dos áreas en los Estados Unidos: reglas éticas y profesionalismo.

Una diferencia sustancial en algunos países, entre ellos Estados Unidos, es que las reglas éticas son examinadas para habilitar en el ejercicio profesional, por lo tanto, la percepción de los estudiantes entre reglas éticas y profesionalismo es distinta ( $\mathrm{Su}$ llivan y otros, 2007). En México no es necesario para todos los estudiantes presentar un examen para acceder al ejercicio de la profesión, aunque en algunos supuestos, de acuerdo con los criterios de las universidades, sí existe un examen que permite la titulación y es administrado por un organismo ajeno a la instituciones educativas. Dicho examen se denomina Examen General para el Egreso de la Licenciatura EGEL. Además, en México las reglas éticas no se examinan más allá de las propias materias que sean impartidas en los planes de estudio que aborden dichas temáticas. Por lo que respecta al profesionalismo, a pesar de estar enunciado en el texto constitucional y en códigos de ética, quienes ejercen la profesión jurídica están poco familiarizados acerca de las acciones precisas en las que se concreta.

Por lo tanto, los trabajos de Hamilton y Montpetit (2007) - que identifican al profesionalismo con siete principios- pueden ayudar a concretar el término profesionalismo que, en un principio, puede parecer un concepto abstracto. Para estos autores, el profesionalismo consiste en: i) estar de acuerdo en cumplir con la ética del deber y los estándares mínimos para la profesión establecidas en las regulaciones, ii) deber esforzarse por realizar, a lo largo de su carrera, la ética de la aspiración —los ideales y valores centrales de la profesión, incluidos la internalización de los más altos estándares para las habilidades profesionales y la conducta ética del abogado-; iii) acordar actuar como un fiduciario en el que su propio interés se ve superado por la devoción al servicio del cliente y el bien público en el área de responsabilidad fiduciaria de la profesión: la justicia; iv) el deber de, a lo largo de su carrera, continuar creciendo en conciencia personal; v) acordar responsabilizar a otros abogados de cumplir con los estándares mínimos establecidos en las reglas y alentarlos a alcanzar tanto los ideales como los valores centrales de la profesión; vi) el deber de dedicar tiempo profesional para servir al bien público, particularmente representando clientes pro bono; y vii) el deber de emprender un compromiso reflexivo continuo, durante su carrera, en la importancia relativa de los ingresos y de la riqueza a la luz de los otros seis principios del profesionalismo.

Por su parte, para Hession (2019), los académicos coinciden en que los componentes básicos del profesionalismo son conocimiento profesional, integridad, honestidad 
e independencia. Hession añade a dichos componentes: cumplimiento de las reglas, competencia emocional, empatía y compasión por los clientes, colegas adversarios y, por sí mismo, ética, competencia, independencia, educación continua, civilidad, servicio pro bono, tutela civil y altruismo público, referentes al Estado de derecho y al sistema de justicia.

En lo que respecta a dicho concepto, en México el Código de Ética del Poder Judicial de la Federación establece que el profesionalismo: «Es la disposición para ejercer, de manera responsable y seria, la función jurisdiccional, con relevante capacidad y aplicación». ${ }^{2}$ En el artículo 4, el código enumera 19 conductas en las que se concreta el profesionalismo en la labor judicial:

4.1. Se abstiene de cualquier acto que pueda mermar la respetabilidad propia de su cargo, tanto en el ámbito público como en el privado.

4.2. Actualiza permanentemente sus conocimientos jurídicos estudiando los precedentes y la jurisprudencia, los textos legales, sus reformas y la doctrina relativa.

4.3. Procura constantemente acrecentar su cultura en las ciencias auxiliares del derecho.

4.4. Estudia con acuciosidad los expedientes y los proyectos en los que deba intervenir.

4.5. Funda y motiva sus resoluciones, evitando las afirmaciones dogmáticas.

4.6. Dedica el tiempo necesario para el despacho expedito de los asuntos de su juzgado o tribunal.

4.7. Asume responsable y valerosamente las consecuencias de sus decisiones.

4.8. Acepta sus errores y aprende de ellos para mejorar su desempeño.

4.9. Guarda celosamente el secreto profesional.

4.10. Lleva a cabo por sí mismo las funciones inherentes e indelegables de su cargo.

4.11. Trata con respeto y consideración a sus subalternos.

4.12. Escucha con atención y respeto los alegatos verbales que le formulen las partes.

4.13. Trata con amabilidad y respeto a los justiciables.

4.14. Administra con diligencia, esmero y eficacia el órgano jurisdiccional a su cargo.

4.15. Cumple puntualmente con el deber de asistir a su tribunal o juzgado.

4.16. Sabe llevar el cumplimiento de su deber hasta el límite de sus posibilidades, y separarse de su cargo cuando su estado de salud u otros motivos personales no le permiten desempeñar eficientemente sus funciones.

4.17. Se abstiene de emitir opiniones sobre la conducta de sus pares.

4.18. Cumple con sus deberes de manera ejemplar para que los servidores públicos a su cargo lo hagan de la misma manera en los que les correspondan.

4.19. Busca con afán que sus acciones reflejen la credibilidad y confianza propias.

2. Suprema Corte de Justicia de la Nación, «Código de Ética del Poder Judicial de la Federación», 2004. 
Del listado se desprenden conductas de diversa índole, muchas de ellas aplicables no tan solo para los profesionales que forman parte del Poder Judicial. Por ejemplo, tres de las diecinueve conductas descritas hacen referencia al estudio y actualización (4.2, 4.3 y 4.4). Por otro lado, otras conductas de las que se describen hacen referencia a la relación que tenga el profesional con subalternos, con las partes en el proceso o con los pares. Por lo que se refiere a los subalternos, los puntos 4.11 y 4.18; por lo que corresponde a los pares, el 4.17. Referente a los justiciables, el 4.12 y el 4.13. El punto 4.1 hace referencia a que cada acto, en el ámbito público y privado, no merme la respetabilidad del cargo. En sentido estricto, los siguientes puntos se refieren a las actividades de la labor jurisdiccional: 4.1, 4.5, 4.6, 4.10, 4.11, 4.13, 4.15, 4.16 y 4.18. Sin embargo, pueden llevarse dichas conductas a otros ámbitos del ejercicio de la profesión jurídica.

Además del texto constitucional y del Código de Ética Judicial del Poder Judicial de la Federación, otra fuente para comprender el alcance del profesionalismo son los códigos de ética, que elaboran los propios profesionales. Dichos códigos muestran los ideales que tienen un grupo de profesionales, las máximas éticas que orientan su conducta y si existen sanciones para quienes quebrantan las normas éticas. En este sentido, los códigos de ética en México contienen las normas éticas a las que hace referencia Sullivan. Además, son un referente para entender el profesionalismo, con la peculiaridad de que los profesionales libremente deciden adherirse a tales normas. Tomando como ejemplo de tales códigos éticos, el Código de Ética Profesional de la Barra Mexicana, Colegio de Abogados (BMA), el del Ilustre y Nacional Colegio de Abogados de México (INCAM) o el de la Asociación Nacional de Abogados de Empresa (ANADE), ninguno de los tres hace referencia, de manera expresa, al profesionalismo. Sin embargo, las conductas que son descritas en el Código de Ética Profesional del Poder Judicial de la Federación como profesionalismo aparecen, en la mayoría de los casos, en los códigos de ética de los colegios de profesionales señalados. No obstante, aparecen bajo otro título.

\section{La enseñanza del profesionalismo}

Aunque el término profesionalismo aparece en el texto constitucional y en el Código de Ética del Poder Judicial de la Federación, en México no es claro en qué conductas precisas se traduce el término. En Estados Unidos, el profesionalismo es uno de los tres aprendizajes en las escuelas de derecho (Sullivan y otros, 2007). Los otros dos son: aprendizaje intelectual o cognitivo, y aprendizaje práctico. Por lo tanto, una primera tarea para las escuelas de derecho en México es posicionar al profesionalismo al lado de los conocimientos teóricos y prácticos, por lo menos, entendiéndolo en el sentido al que hemos hecho referencia al principio de este trabajo. Considerar el profesionalismo dentro de la enseñanza requiere de que los estudiantes entiendan y 
demuestren una conducta de acuerdo con tal profesionalismo, y que posean el conjunto de habilidades necesarias para continuar desarrollando la identidad profesional a lo largo de su carrera (O'Sullivan y otros, 2012). Un obstáculo en la enseñanza del profesionalismo en México ha sido la falta de claridad acerca del término y la escasa reflexión acerca de la propia identidad de los profesionales del derecho, así como del sentido de la profesión. Sin embargo, la experiencia al respecto, en otros países y en otras profesiones, contribuye al proceso de sensibilización en relación con su enseñanza: «professionalism needs to become more explicit and better diffused throughout legal preparation» (Sullivan y otros, 2007). Entonces, será labor de las escuelas de derecho formar a sus estudiantes en tal sentido.

Las escuelas de derecho en México, al profundizar en lo que es el profesionalismo y la forma en cómo se enseña, contribuyen al fortalecimiento de la profesión para que pueda cumplir con sus fines. A fin de cuentas, las escuelas, lo que deben desarrollar son habilidades y competencias que puedan ser útiles para los estudiantes en el futuro (Gatgens, 2020), además de que las escuelas pueden ayudar a la profesión para encontrar formas que le permitan cumplir con su cometido (Sullivan y otros, 2007). «Se confía más, con las competencias, en la capacidad de respuesta ante situaciones variables e imprevisibles que en un catálogo fijado de respuestas deseables y supuestamente valiosas» (Cullen, 1997: 90). De este modo, la noción de competencia no queda reducida a una mera apropiación de conocimientos, sino, por el contrario, abarca todo un conjunto de capacidades que permiten a la persona responsable convertirse en ese «ser competente» para realizar múltiples acciones. Así, la persona competente puede proyectar y evidenciar su «capacidad de resolver un problema dado dentro de un contexto específico y cambiante» (Lapenta, 2020). En este sentido, el profesionalismo tendría que ser asumido en las escuelas.

Por lo tanto, un primer paso es que las escuelas de derecho decidan enseñar el profesionalismo, porque la enseñanza va más allá de lo académico: también refuerza las actividades sociales, la ciudadanía y la resiliencia (Robert, 2018). Y, como un aprendizaje diferente a los conocimientos o habilidades técnicas que requiere el profesional del derecho, es necesario que también se enseñe, se evalúe y se generen evidencias de dicho aprendizaje (O'Sullivan y otros, 2012). Ejemplo de ello es lo que ocurre con los dentistas: de acuerdo con el Consejo General Dental, es necesario que los estudiantes reconozcan la importancia del profesionalismo y puedan demostrar las actitudes profesionales desde el inicio de su formación, además de subraya la enseñanza y evaluación de profesionalismo (Taylor, Grey y Checkland, 2017).

En este mismo sentido, Hamilton y Brabbit (2007) sostienen que el profesionalismo tiene que ser enseñado de forma explícita. Rescatan las aportaciones en las áreas de salud de Rules y Bebeau, para quienes la labor de los educadores es facilitar la propia evaluación y reflexión. Para lograrlo, proponen diversos caminos, entre ellos, Hamilton y Brabbit establecen la necesidad de que los futuros profesionales tengan 
un mentor para interiorizar los principios del profesionalismo y que, así, el estudiante pueda alcanzar sus metas. Por su parte, Sullivan enfatiza en el papel que juegan los modelos a seguir (O'Sullivan y otros, 2012), lo que abre un abanico de posibilidades que las escuelas de derecho pueden explotar.

La enseñanza del profesionalismo, como competencia profesional, presenta diversos retos, uno de ellos es la evaluación. Sin tal evaluación es imposible alcanzar el profesionalismo (Lynch, Surdyk y Eiser, 2004). En este sentido, hay varios modelos. Uno de ellos es seguir con las recomendaciones que hacen los grupos de profesionales. Por ejemplo, para los médicos, el Consejo de Acreditación para la Educación Médica de Graduados de Estados Unidos sugiere diversos métodos de evaluación, entre ellos la evaluación multicéntrica, que comprende la autoevaluación interpersonal, las evaluaciones de pacientes, la autoevaluación y los portafolios (Hernández y otros, 2010).

La experiencia en el ámbito médico muestra que no hay evidencia que permita afirmar que, por sí misma, la formulación de resultados de aprendizaje y la enseñanza del profesionalismo produzca un cambio en las actitudes de los profesionales, de ahí que sea necesaria su evaluación (Goldie, 2013: e952). La evaluación de profesionalismo es un ámbito en constante evolución. Las competencias son tareas que el profesional de la medicina calificado debe ser capaz de hacer exitosamente (Goldie, 2013: e954); lo mismo podemos decir de las competencias del profesional del derecho. La evaluación debe, idealmente, medir actuaciones en el día a día de la práctica (Goldie, 2013: e954). Por lo tanto, un punto de partida puede ser la autoevaluación que hacen los estudiantes de derecho en las acciones que les corresponde realizar día a día desde el inicio de los estudios de licenciatura.

\section{La autoevaluación del profesionalismo en estudiantes de primer año de licenciatura}

En virtud de que el profesionalismo es considerado como una competencia indispensable en el ejercicio de la función jurisdiccional, además de otras áreas de ejercicio, debería ser considerado como un resultado de aprendizaje en las licenciaturas en derecho en México. Tal ha sido el camino que han trazado para la enseñanza del profesionalismo en otros países las áreas de la salud y el derecho, lo que ha obligado a que las escuelas adopten medidas al respecto. Después de ser considerado como resultado de aprendizaje, resulta necesario, en el proceso enseñanza-aprendizaje, la evaluación de los estudiantes. Curcio (2018) propone un plan de cinco pasos para evaluar los resultados de aprendizaje que la American Bar Association ha determinado para las escuelas de derecho en Estados Unidos: i) desarrollar una rúbrica; ii) elegir el curso en el que se aplicará la rúbrica; iii) calificar y evaluar la rúbrica; iv) ingresar datos; y v) utilizar.

En México, los organismos que acreditan de forma voluntaria a la educación ju- 
rídica no señalan cuáles son los resultados de aprendizaje esperados. Sin embargo, pueden los propios docentes o las instituciones de educación superior llevar a cabo esfuerzos que permitan que el profesionalismo sea enseñado. El proceso que Curcio (2018) propone es un camino que puede seguirse en México para enseñar el profesionalismo entre los estudiantes mediante rúbricas existentes y el desarrollo futuro de otras. Por otra parte, la literatura sugiere que la evaluación del profesionalismo debe ser a través de diferentes métodos (Lynch, Surdyk y Eiser, 2004), por lo que la autoevaluación es uno de ellos. En tal sentido, en esta investigación se utilizó la rúbrica elaborada por el Holloran Center for Ethical Leadership in the Professions (Holloran Center for Ethical Leadership in the Professions, 2019), que forma parte del Holloran Competency Milestone (Holloran Center for Ethical Leadership in the Professions, 2020), proyecto que consiste en el desarrollo de rúbricas que describen varias etapas de desarrollo de una competencia o subcompetencia asociada con un resultado de aprendizaje de los estudiantes de derecho en Estados Unidos. En la rúbrica utilizada se aborda un aspecto del profesionalismo: cumplimiento de acuerdos. El cumplimiento de acuerdos es una de las subcompetencias del profesionalismo esenciales para el ejercicio de la profesión jurídica.

Los participantes de la investigación fueron estudiantes que cursaban el primer grado de la licenciatura en Derecho en agosto de 2019 en una universidad del occidente de México. La literatura sugiere que la evaluación de profesionalismo debe iniciar pronto (Lynch, Surdyk y Eiser, 2004). La autoevaluación fue hecha por 52 alumnos de forma anónima durante el primer día de clases. Los estudiantes que resolvieron el cuestionario disponible de forma electrónica lo hicieron dentro de la materia de Introducción al Estudio del Derecho el primer día de clases del programa de licenciatura. El ejercicio de autoevaluación tuvo por objetivo que los alumnos conocieran en qué consistía el profesionalismo con respecto al cumplimiento de acuerdos y, así, pudieran conocer el grado de desarrollo de dicha competencia al momento de iniciar sus estudios universitarios. No se presentó ningún obstáculo para que los alumnos pudieran autoevaluarse.

El mapa curricular de la licenciatura está compuesto por diez semestres, y en el último semestre está la materia de Ética Profesional. Sin embargo, la adquisición del profesionalismo como competencia no es tarea de una materia o un esfuerzo aislado, como ha sido señalado. La materia de Introducción al Estudio del Derecho en dicha universidad tiene, entre otros, el objetivo de presentar a los futuros profesionales los ideales que tiene la profesión y las competencias que se deben desarrollar para alcanzar tales ideales.

La rúbrica utilizada, que forma parte del Holloran Competency Milestone, contempla cinco etapas de desarrollo de la subcompetencia: novato, principiante avanzado, medio, competente o excepcional (Holloran Center for Ethical Leadership in the Professions, 2020). 
De acuerdo con la rúbrica, los alumnos se autoevaluaron en función de diez criterios:

1. Uso de calendario y sistema de gestión de proyectos.

2. Puntualidad o ausencia a clases y reuniones.

3. Aviso de puntualidad o ausencia a clases y reuniones.

4. Entrega de trabajo en tiempo.

5. Aviso de reentrega de trabajo en tiempo.

6. Preparación para la clase.

7. Aviso de repreparación para la clase.

8. Participación en la clase.

9. Uso de tecnología durante la clase.

10. Compromiso con la excelencia y el trabajo de alta calidad.

El objetivo que orientó esta investigación fue responder a la pregunta: ¿De qué forma los alumnos podrían conocer el nivel de profesionalismo en el que se encuentran? Por otro lado, también resultó importante mostrar a alumnos de primer semestre todas las fases de desarrollo de la competencia. La evaluación del profesionalismo resulta necesaria, aunque no existe un método único para llevarla a cabo (Arnold, 2002). Resulta indispensable que la competencia de profesionalismo que se requerirá en la vida profesional y a la que se hace referencia en la Constitución se desarrolle durante los años de educación en la licenciatura.

En lo que respecta a los resultados en esta investigación, se identificó que la mayor parte de los alumnos, de acuerdo con los diez criterios evaluados, se ubican en un nivel medio (tabla 1, tabla 2 y tabla 3), toda vez que la mayor parte de los alumnos se ubica en cuatro criterios: participación en la clase, el aviso de repreparación para la clase, uso de la tecnología durante la clase, compromiso con la excelencia y el trabajo de alta calidad. En el mismo nivel medio se ubicaron, en segundo lugar, los criterios de uso de calendario y el sistema de gestión de proyectos, la puntualidad o ausencia de clases y reuniones, el aviso de puntualidad o ausencia a clases y reuniones, y la entrega de trabajo en tiempo.

Una vez analizadas las respuestas, se obtuvo como resultado que los alumnos se autoevaluaron en la fase de desarrollo excepcional solo en un criterio: aviso de reentrega de trabajo en tiempo. Por otra parte, con respecto al criterio puntualidad o ausencia a clases y reuniones, los alumnos se evaluaron en las fases de desarrollo más alta: la mayoría de ellos se evaluó en un nivel competente y, en segundo lugar, en nivel excepcional. 
Tabla 1. Uso de calendario, puntualidad y aviso

\begin{tabular}{|c|c|c|c|c|c|c|}
\hline & \multicolumn{2}{|c|}{$\begin{array}{l}\text { Uso de calendario y sistema } \\
\text { de gestión de proyectos }\end{array}$} & \multicolumn{2}{|c|}{$\begin{array}{l}\text { Puntualidad/ausencia } \\
\text { en clases y reuniones }\end{array}$} & \multicolumn{2}{|c|}{$\begin{array}{c}\text { Aviso de puntualidad/ausencia } \\
\text { a clases y reuniones }\end{array}$} \\
\hline & Fases & Conteo & Fases & Conteo & Fases & Conteo \\
\hline Novato & Aún no desarrollado & $21,2 \%$ & $\begin{array}{l}\text { Con frecuencia llega tarde } \\
\text { a clases y reuniones }\end{array}$ & $0 \%$ & $\begin{array}{l}\text { Usualmente no ofrece } \\
\text { un aviso en tiempo ni } \\
\text { proporciona explicaciones } \\
\text { si llega tarde o no asistirá }\end{array}$ & $1,9 \%$ \\
\hline $\begin{array}{l}\text { Principiante } \\
\text { avanzado }\end{array}$ & Sistemas en desarrollo & $32,7 \%$ & $\begin{array}{l}\text { Ocasionalmente llega } \\
\text { en tiempo a clases y re- } \\
\text { uniones }\end{array}$ & $5,8 \%$ & $\begin{array}{l}\text { Ocasionalmente ofrece } \\
\text { un aviso en tiempo } 0 \\
\text { proporciona explicaciones } \\
\text { si llegará tarde o no } \\
\text { asistirá }\end{array}$ & $3,9 \%$ \\
\hline Medio & $\begin{array}{l}\text { Sistemas funcionales } \\
\text { desarrollados y probados }\end{array}$ & $30,8 \%$ & $\begin{array}{l}\text { Usual o frecuentemente } \\
\text { asiste a clases y reuniones } \\
\text { en tiempo }\end{array}$ & $11,5 \%$ & $\begin{array}{l}\text { Usual o frecuentemente } \\
\text { ofrece un aviso en tiempo } \\
\text { o da una explicación } \\
\text { cuando llegará tarde o no } \\
\text { asistirá }\end{array}$ & $26,9 \%$ \\
\hline Competente & $\begin{array}{l}\text { Excelentes sistemas desa- } \\
\text { rrollados y probados }\end{array}$ & $15,4 \%$ & $\begin{array}{l}\text { Siempre o casi siempre } \\
\text { llega a clases y reuniones } \\
\text { en tiempo }\end{array}$ & $44,2 \%$ & $\begin{array}{l}\text { Siempre o casi siempre } \\
\text { ofrece un aviso en tiempo } \\
\text { o explicación cuando } \\
\text { llegará tarde o no asistirá }\end{array}$ & $51,9 \%$ \\
\hline Excepcional & $\begin{array}{l}\text { Sistemas funcionales } \\
\text { desarrollados y probados } \\
\text { en la práctica }\end{array}$ & $0 \%$ & $\begin{array}{l}\text { Siempre llega a clases y } \\
\text { reuniones en tiempo }\end{array}$ & $38,5 \%$ & $\begin{array}{l}\text { Siempre llega a clases y a } \\
\text { reuniones en tiempo }\end{array}$ & $15,4 \%$ \\
\hline
\end{tabular}

Se evidenció, de acuerdo con los resultados, que en seis de los criterios evaluados no hay alumnos que se identifiquen en la fase de novatos. Dichos criterios son: puntualidad o ausencia a clases y reuniones, entrega de trabajo en tiempo, aviso de reentrega de trabajo en tiempo, preparación para la clase, participación en clase y compromiso con la excelencia, y el trabajo de alta calidad (tabla 1, tabla 2 y tabla 3 ).

De acuerdo con el análisis y la interpretación de los datos, el criterio en el que se autoevalúan en las fases más bajas de desarrollo es el uso de calendario y en la gestión de proyectos; el porcentaje más alto de alumnos se autoevaluó como principiante avanzado; y, en segundo lugar del porcentaje más alto, fueron los estudiantes que se identifican con el nivel medio.

Futuras investigaciones podrían evaluar las fases de desarrollo de las competencias en otros momentos de la formación profesional, para evaluar si efectivamente, durante el proceso de formación universitaria, han desarrollado la competencia. Por otra parte, a la autoevaluación se podría sumar otro tipo de evaluaciones, entre ellas: la evaluación por pares. 
Tabla 2. Entrega de trabajo en tiempo, preparación para clase y avisos

\begin{tabular}{|c|c|c|c|c|c|c|c|c|}
\hline & \multicolumn{2}{|c|}{$\begin{array}{c}\text { Entrega de trabajo en } \\
\text { tiempo }\end{array}$} & \multicolumn{2}{|c|}{$\begin{array}{l}\text { Aviso de reentrega de } \\
\text { trabajo en tiempo }\end{array}$} & \multicolumn{2}{|c|}{ Preparación para la clase } & \multicolumn{2}{|c|}{$\begin{array}{c}\text { Aviso de repreparación } \\
\text { para la clase }\end{array}$} \\
\hline & Fases & Conteo & Fases & Conteo & Fases & Conteo & Fases & Conteo \\
\hline Novato & $\begin{array}{l}\text { Con frecuencia } \\
\text { pierde horarios y } \\
\text { fechas límite }\end{array}$ & $0 \%$ & $\begin{array}{l}\text { Con frecuencia no } \\
\text { ofrece un aviso o } \\
\text { explicación cuando } \\
\text { entrega un encargo } \\
\text { o trabajo fuera de } \\
\text { tiempo }\end{array}$ & $0 \%$ & $\begin{array}{l}\text { Usualmente no se } \\
\text { prepara para clases } \\
\text { y reuniones }\end{array}$ & $0 \%$ & $\begin{array}{l}\text { Con frecuencia no } \\
\text { ofrece un aviso en } \\
\text { tiempo ni expli- } \\
\text { caciones cuando } \\
\text { no se encuentra } \\
\text { preparado }\end{array}$ & $5.8 \%$ \\
\hline $\begin{array}{l}\text { Princi- } \\
\text { piante } \\
\text { avanzado }\end{array}$ & $\begin{array}{l}\text { 0casionalmente } \\
\text { pierde horarios y } \\
\text { fechas límite }\end{array}$ & $3,9 \%$ & $\begin{array}{l}\text { Ocasionalmente } \\
\text { ofrece en tiempo } \\
\text { un aviso cuando } \\
\text { entrega un encargo } \\
\text { o trabajo fuera de } \\
\text { tiempo }\end{array}$ & $9,6 \%$ & $\begin{array}{l}\text { Ocasionalmente se } \\
\text { prepara para clases } \\
\text { y reuniones }\end{array}$ & $21,2 \%$ & $\begin{array}{l}\text { Ocasionalmente } \\
\text { ofrece un aviso } \\
\text { en tiempo o propor- } \\
\text { ciona explicaciones } \\
\text { cuando no se en- } \\
\text { cuentra preparado }\end{array}$ & $15,4 \%$ \\
\hline Medio & $\begin{array}{l}\text { Raramente pierde } \\
\text { horarios y fechas } \\
\text { límites }\end{array}$ & $28,6 \%$ & $\begin{array}{l}\text { Siempre entrega a } \\
\text { tiempo dentro del } \\
\text { plazo establecido } \\
\text { anticipando la } \\
\text { posibilidad de } \\
\text { inconvenientes } \\
\text { inesperados como } \\
\text { precaución }\end{array}$ & $38,5 \%$ & $\begin{array}{l}\text { Usual o frecuente- } \\
\text { mente se encuentra } \\
\text { preparado para } \\
\text { clases y reuniones }\end{array}$ & $42,3 \%$ & $\begin{array}{l}\text { Usual o frecuente- } \\
\text { mente ofrece un } \\
\text { aviso en tiempo } 0 \\
\text { explicación cuando } \\
\text { no se encuentra } \\
\text { preparado }\end{array}$ & $55,8 \%$ \\
\hline $\begin{array}{l}\text { Compe- } \\
\text { tente }\end{array}$ & $\begin{array}{l}\text { Siempre entrega a } \\
\text { tiempo dentro del } \\
\text { plazo establecido } \\
\text { anticipando la } \\
\text { posibilidad de } \\
\text { inconvenientes } \\
\text { inesperados como } \\
\text { precaución }\end{array}$ & $59,6 \%$ & $\begin{array}{l}\text { Siempre entrega } \\
\text { tareas elaboradas } \\
\text { más allá de lo } \\
\text { solicitado. Excede } \\
\text { las expectativas }\end{array}$ & $7,7 \%$ & $\begin{array}{l}\text { Siempre se encuen- } \\
\text { tra preparado para } \\
\text { clases y reuniones }\end{array}$ & $32,7 \%$ & $\begin{array}{l}\text { Siempre se encuen- } \\
\text { tra preparado para } \\
\text { clases y reuniones }\end{array}$ & $23,1 \%$ \\
\hline $\begin{array}{l}\text { Excepcio- } \\
\text { nal }\end{array}$ & $\begin{array}{l}\text { Siempre entrega } \\
\text { tareas elaboradas } \\
\text { más allá de lo } \\
\text { solicitado. Excede } \\
\text { las expectativas }\end{array}$ & $7,7 \%$ & $\begin{array}{l}\text { Usual o frecuente- } \\
\text { mente ofrece un } \\
\text { aviso en tiempo } 0 \\
\text { explicación cuando } \\
\text { entrega un encargo } \\
\text { o trabajo fuera de } \\
\text { tiempo }\end{array}$ & $44,2 \%$ & $\begin{array}{l}\text { Siempre se encuen- } \\
\text { tra preparado para } \\
\text { clases y reuniones. } \\
\text { Excede las expec- } \\
\text { tativas }\end{array}$ & $3,9 \%$ & $\begin{array}{l}\text { Siempre se encuen- } \\
\text { tra preparado para } \\
\text { clases y reuniones. } \\
\text { Excede las expec- } \\
\text { tativas }\end{array}$ & $0 \%$ \\
\hline
\end{tabular}


Tabla 3. Participación en clase, uso de tecnología, compromiso con la excelencia y el trabajo de alta calidad

\begin{tabular}{|c|c|c|c|c|c|c|}
\hline & \multicolumn{2}{|c|}{ Participación en la clase } & \multicolumn{2}{|l|}{$\begin{array}{l}\text { Uso de tecnología } \\
\text { durante la clase }\end{array}$} & \multicolumn{2}{|c|}{$\begin{array}{c}\text { Compromiso con la excelencia } \\
\text { y el trabajo de alta calidad }\end{array}$} \\
\hline & Fases & Conteo & Fases & Conteo & Fases & Conteo \\
\hline Novato & $\begin{array}{l}\text { La participación en } \\
\text { clases con frecuencia no es } \\
\text { suficiente }\end{array}$ & $0 \%$ & $\begin{array}{l}\text { Usualmente no utiliza } \\
\text { tecnología en clase } \\
\text { apropiadamente }\end{array}$ & $1,9 \%$ & $\begin{array}{l}\text { Con frecuencia entrega un } \\
\text { trabajo de baja calidad }\end{array}$ & $0 \%$ \\
\hline $\begin{array}{l}\text { Principiante } \\
\text { avanzado }\end{array}$ & $\begin{array}{l}\text { La participación en clases } \\
\text { o reuniones ocasional- } \\
\text { mente se encuentra de } \\
\text { acuerdo con el mínimo } \\
\text { requerido }\end{array}$ & $13,5 \%$ & $\begin{array}{l}\text { Ocasionalmente utiliza } \\
\text { tecnología en clase apro- } \\
\text { piadamente }\end{array}$ & $11,5 \%$ & $\begin{array}{l}\text { Usualmente entrega un } \\
\text { trabajo de baja calidad }\end{array}$ & $0 \%$ \\
\hline Medio & $\begin{array}{l}\text { Su participación en clases } \\
\text { o reuniones corresponde } \\
\text { al mínimo requerido }\end{array}$ & $44,2 \%$ & $\begin{array}{l}\text { Raramente utiliza tecnolo- } \\
\text { gía en clases apropiada- } \\
\text { mente }\end{array}$ & $15,4 \%$ & $\begin{array}{l}\text { Raramente entrega un } \\
\text { trabajo de baja calidad }\end{array}$ & $48,1 \%$ \\
\hline Competente & $\begin{array}{l}\text { Su participación en clases } \\
\text { o reuniones excede algu- } \\
\text { nas veces las expectativas }\end{array}$ & $32,7 \%$ & $\begin{array}{l}\text { Siempre o casi siempre } \\
\text { utiliza tecnología en clase } \\
\text { apropiadamente }\end{array}$ & $50 \%$ & $\begin{array}{l}\text { Regularmente entrega } \\
\text { tareas elaboradas más allá } \\
\text { de lo solicitado. Excede las } \\
\text { expectativas }\end{array}$ & $5,8 \%$ \\
\hline Excepcional & $\begin{array}{l}\text { Su participación en clases } \\
\text { y reuniones usualmente } \\
\text { excede las expectativas }\end{array}$ & $9,6 \%$ & $\begin{array}{l}\text { Siempre utiliza tecnología } \\
\text { en clase apropiadamente }\end{array}$ & $21,2 \%$ & $\begin{array}{l}\text { Siempre entrega trabajos } \\
\text { de alta calidad }\end{array}$ & $46,2 \%$ \\
\hline
\end{tabular}

\section{Conclusiones}

Esta investigación ha mostrado que, a pesar de que el profesionalismo aparece en el texto constitucional, en México todavía ni su significado ni alcance son claros. El Código de Ética del Poder Judicial de la Federación detalla algunas conductas con respecto al profesionalismo, sin embargo, los códigos de los colegios de abogados no hacen referencia a dicho término. En este sentido, a través de la investigación bibliográfica se ha mostrado que el profesionalismo ha sido un tema de interés al momento de estudiar las profesiones.

La sociología destaca la importancia del término para configurar la identidad de los profesionales. Por otra parte, por lo que corresponde a los profesionales del derecho, es importante aclarar el alcance del término. En otras áreas, entre ellas las áreas de la salud, existe información que permite a los futuros profesionales entender el alcance del término. Por su parte, las escuelas de derecho juegan un papel importante para formar en este sentido a los profesionales. Por un lado, formarlos en profesionalismo requiere que este sea reconocido como una competencia profesional. Por otro, que se desarrollen evaluaciones que permitan que sea valorado el nivel de desarrollo al respecto. 
En este trabajo, se realizó un ejercicio de autoevaluación entre estudiantes de primer año de licenciatura utilizando las rúbricas del Holloran Competency Milestone. Dicho ejercicio permitió que los estudiantes se familiarizaran con el término profesionalismo y pudieran autoevaluarse en la subcompetencia de cumplimiento de acuerdos. A partir de dicho ejercicio, los alumnos conocerán algunos aspectos del profesionalismo. Futuras investigaciones podrán evaluar el nivel de profesionalismo en otros momentos de la educación jurídica y conocer en qué grado se ha desarrollado al momento de concluir los estudios de licenciatura. Además, futuras investigaciones podrían llevarse a cabo midiendo el grado de desarrollo de profesionalismo entre quienes ya ejercen el derecho. Sumado a lo anterior, los ejercicios de autoevaluación podrían complementarse con otras evaluaciones realizadas por los profesores y otros estudiantes. Si se desea que los futuros profesionales desarrollen sus tareas de la mejor forma, la educación jurídica es el período idóneo para entrenarlos en tal sentido.

\section{Referencias}

Aguado, David, Antonio, Marta Antúnez González y Teresa de Dios (2017). «Evaluación de competencias transversales en universitarios. propiedades psicométricas iniciales del cuestionario de competencias transversales». REICE: Revista Iberoamericana Sobre Calidad, Eficacia y Cambio En Educación, 15 (2): 129-152. DOI: 10.15366/reice2017.15.2.007.

ARNOLD, Louise (2002). «Assessing professional behavior: Yesterday, today, and tomorrow». Academic Medicine, 6 (77): 502-515. DOI: 10.1097/00001888-200206000-00006.

BAGUST, Joanne (2013). «The legal profession and the business of law». Sydney Law Review, 27 (35): 27-52. Disponible en https://bit.ly/3muhbL3.

Bas PeÑa, Encarna, Elvira Ferre Jaén y Antonio Maurandi López (2017). «Educación Superior, competencias transversales y género: Validación de un cuestionario». Revista de Humanidades, 31: 57-76. Disponible en https://bit.ly/3EkF2TD.

Bowern, Michael y Oliver K. Burmeister (2013). «Business benefits from keeping codes of ethics up to date». En John Weckert y Richard Lucas (compiladores), Professionalism in the information and communication technology industry (pp. 295-311). Canberra: ANU Press.

Cruess, Sylvia y Richard Cruess (2016). «Professionalism as a social construct: The evolution of a concept». Journal of Graduate Medical Education, 2 (8): 265-267. Disponible en https://bit.ly/3ySBirx.

Cullen, Carlos (1997). Crítica de las razones de educar. Temas de la filosofía de la educación. Buenos Aires: Paidós.

Curcio, Andrea A. (2018). «A simple low-cost institutional learning-outcomes as- 
sessment process». Journal of Legal Education, 67 (2): 489-530. Disponible en https://bit.ly/3em3Zn2.

Evetts, Julia (2013). «Professionalism: Value and ideology». Current Sociology, 5-6 (61): 778-796. DOI: 10.1177/o011392113479316.

Francis, Andrew M. (2005). «Legal ethics, the marketplace and the fragmentation of legal professionalism». International Journal of the Legal Profession, 2 (12): 173200. DOI: 10.1080/09695950500226623.

Frye, Victoria, Marlene Camacho, Kaliris Salas, Tashuna Albritton, Darwin Deen, Nancy Sohler, Samantha Barrick, Joao Nunes (2020). «Professionalism: The wrong tool to solve the right problem?». Academic Medicine, 6 (95): 860-863. DOI: 10.1097/ACM.0000000000003266.

Gatgens, Erick (2020). «Reflexiones pedagógicas sobre la enseñanza del derecho». Revista de la Universidad de Costa Rica, 2 (44): 559-569 Disponible en https://bit. ly/3EpxMGf.

GolDIE, John (2013). «Assessment of professionalism: A consolidation of current thinking». Medical Teacher, 35 (2): e952-e956. DOI: 10.3109/0142159X.2012.714888.

GonzÁlez Ramos, Rosalba Georgina (2017). «Exploración de planes de estudios de escuelas de Derecho y el desafío de cambio al enfoque por competencias». Academia. Revista sobre Enseñanza del Derecho de Buenos Aires, 30 (15): 129-152. Disponible en https://bit.ly/3mwiRUm.

Hamilton, Neil (2012). «Too few examples, too much law». Academic Questions, 3 (25): 356-36o. DOI: 10.1007/s12129-012-9299-2.

-. (2017a). «Formation-of-an-ethical-professional-identity (professionalism) learning outcomes and e-portfolio formative assessments». The University of the Pacific Law Review, 4 (48): 847-873. Disponible en https://bit.ly/3FqZRoX.

-. (2017b). «Professional-identity/professional-formation/professionalism learning outcomes: What we learn about assessment from medical education?». University of St. Thomas Law Journal, 14 (2). Disponible en https://bit.ly/3JaKZpU.

Hamilton, Neil y Lisa Montpetit Brabbit (2007). «Fostering professionalism through mentoring». Journal of Legal Education, 1 (57): 102-129. Disponible en https://bit. ly/3qfQGKy.

Hernández, Claudia, Julio Hernández, Lui González y Salim Barquet (2010). «Formación en ética y profesionalismo para las nuevas generación de médicos». Persona y Bioética, 1 (14): 30-39. Disponible en https://bit.ly/3elLjUI.

Hession, Rachael (2019). «Professionalism in the legal profession-can you teach it? A phenomenographic study of Irish legal education stakeholders' perceptions». The Law Teacher, 3 (53): 245-262. DOI: 10.1080/03069400.2018.1503519.

Holloran Center for Ethical Leadership in the Professions (2019). «Assessment of student's demonstration of professionalism: Honoring commitments» Disponible en https://bit.ly/3moGn5R. 
LAPENTA, Lucía Irene (2020). «Una invitación a pensar sobre competencias docentes en las Facultades de Derecho». Revista Pedagogía Universitaria y Didáctica del Derecho, 7 (2): 231-246. DOI: 10.5354/0719-5885.2020.57911.

LynCH, Deirdre, Patricia Surdyk y Arnold Eiser (2004). "Assessing professionalism: A review of the literature». Medical Teacher, 4 (26): 366-373. DOI: 10.1080/01421590410001696434.

MoORHEAD, Richard (2014). «Precarious professionalism: Some empirical and behavioural perspectives on lawyers». Current Legal Problems, 1 (67): 447-481. DOI: $10.1093 / \mathrm{clp} /$ cuuoo 4 .

Olson, G., y Lazaretti, I. K. (2019). «A transformação da educação jurídica no seculo XXI: A formação das competências profissionais dos operadores do direito». Revista de Pesquisa e Educação Jurídica, 2 (2): 193-213. Disponible en https://bit. ly/3ehxxls.

O'Sullivan, Helen, Walther Van Mook, Ray Fewtrell y Val Wass (2012). «Integrating professionalism into the curriculum». Medical Teacher, 2 (34): 155-157. DOI: 10.3109/0142159X.2011.595600.

Robert, Bruno (2018). «When Did the U.S. Stop Seeing Teachers as Professionals?». Harvard Business Review. Disponible en https://bit.ly/3FociuD.

SAldaña, Javier (2014). «Profesionalismo: Principio básico de la ética judicial» En Juan Vega (editor), Temas Selectos de derecho internacional privado y de derechos humanos. Estudios en homenaje a Sonia Rodríguez Jiménez (pp. 415-445). Ciudad de México: UNAM.

Sullivan, William, Anne Colby, Judith W. Wegner, Lloyd Bond y Lee S.Shulman (2007). Educating lawyers: Preparation for the profession o law. San Francisco: Jossey-Bass.

TAYLOR, Carly, Nicholas Grey y Katherine Checkland (2017). «Professionalism... it depends where you're standing». British Dental Journal, 222: 889-892. DOI: 10.1038/sj.bdj.2017.502.

WEGNER, Judith Welch (2018). «Law school assessment in the context of accreditation: Critical questions, what we know and don't know, and what we should do next». Journal of Legal Education, 67 (2): 412-461. Disponible en https://bit. ly/3EjdIVL.

\section{Sobre las autoras}

YURIXHI GaLlardo es profesora investigadora del Instituto de Humanidades en la Universidad Panamericana. Doctora en Derecho por la Universidad Panamericana, campus Guadalajara; maestra en Historia del Pensamiento por la Universidad Panamericana, campus Ciudad de México; y maestra en Estudios Humanísticos y Sociales por la Universitat Abat Oliba, CEU. También es miembro del Sistema Nacional de 
Investigadores SNI-I de México. Su correo electrónico es ygallard@up.edu.mx. (D) https://orcid.org/oooo-0003-1365-0179.

Montserrat Gómez Villaseñor es estudiante de Licenciatura en Derecho en la Universidad Panamericana. Su correo electrónico es 0214489@up.edu.mx. (D) https:// orcid.org/00oo-0002-6546-504X. 
La Revista Pedagogía Universitaria y Didáctica del Derecho (RPUDD) es una publicación científica semestral que contribuye a la reflexión multidisciplinaria sobre pedagogía universitaria y didáctica del derecho, para la formación y consolidación de esta área de investigación; así como a la difusión de prácticas innovadoras en la enseñanza-aprendizaje del derecho considerando el contexto nacional e internacional. Es una publicación electrónica internacional con una codirección entre Brasil y Chile.

\author{
DIRECTORA \\ María Francisca Elgueta Rosas \\ Universidad de Chile \\ DIRECTOR \\ Renato Duro Dias \\ Universidad Federal de Rio Grande, Brasil \\ SITIO WEB \\ pedagogiaderecho.uchile.cl \\ CORREO ELECTRÓNICO \\ rpedagogia@derecho.uchile.cl \\ LICENCIA DE ESTE ARTÍCULO
}

Creative Commons Atribución Compartir Igual 4.o Internacional

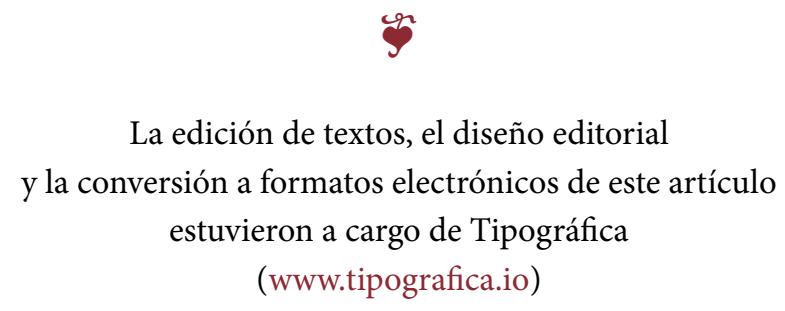

